ARTICLE

DOI: $10.1038 / \mathrm{s} 41467-018-06514-5$

\title{
Identification of small-molecule ion channel modulators in C. elegans channelopathy models
}

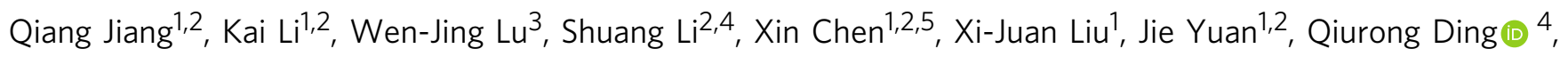
Feng $\operatorname{Lan}^{3} \&$ Shi-Qing Cai ${ }^{1}$

Ion channels are important therapeutic targets, but the discovery of ion channel drugs remains challenging due to a lack of assays that allow high-throughput screening in the physiological context. Here we report $C$. elegans phenotype-based methods for screening ion channel drugs. Expression of modified human ether-a-go-go-related gene (hERG) potassium channels in C. elegans results in egg-laying and locomotive defects, which offer indicators for screening small-molecule channel modulators. Screening in worms expressing hERGA561V, which carries a trafficking-defective mutation A561V known to associate with long-QT syndrome, identifies two functional correctors Prostratin and ingenol-3,20-dibenzoate. These compounds activate $\mathrm{PKC} \varepsilon$ signaling and consequently phosphorylate $\mathrm{S} 606$ at the pore region of the channel to promote hERGA561V trafficking to the plasma membrane. Importantly, the compounds correct electrophysiological abnormalities in hiPSC-derived cardiomyocytes bearing a heterozygous CRISPR/Cas9-edited hERGA561V. Thus, we have developed an in vivo high-throughput method for screening compounds that have therapeutic potential in treating channelopathies.

\footnotetext{
${ }^{1}$ Institute of Neuroscience and State Key Laboratory of Neuroscience, CAS Center for Excellence in Brain Science and Intelligence Technology, Shanghai Institutes for Biological Sciences, Chinese Academy of Sciences, 200031 Shanghai, China. ${ }^{2}$ University of Chinese Academy of Sciences, 100049 Beijing, China. ${ }^{3}$ Beijing Laboratory for Cardiovascular Precision Medicine, Beijing Anzhen Hospital, Capital Medical University, 100029 Beijing, China. ${ }^{4}$ CAS Key Laboratory of Nutrition, Metabolism and Food Safety, Shanghai Institute of Nutrition and Health, Shanghai Institutes for Biological Sciences, Chinese Academy of Sciences, Shanghai 200031, China. ${ }^{5}$ Present address: Developmental and Stem Cell Program, Arthur and Sonia Labatt Brain Tumor Research Centre, Hospital for Sick Children, Toronto M5G 1X8 ON, Canada. These authors contributed equally: Qiang Jiang, Kai Li. Correspondence and requests for materials should be addressed to F.L. (email: fenglan@ccmu.edu.cn) or to S.-Q.C. (email: sqcai@ion.ac.cn)
} 
on channels are the molecular basis for the cell excitability. Malfunction of ion channels causes over 55 different inherited human diseases known as channelopathies ${ }^{1}$. Although $15 \%$ of US Food and Drug Administration (FDA)-approved drugs act through targeting ion channels ${ }^{2,3}$, there remains an urgent need for developing drugs for many untreated channelopathies. Discovery of new drugs targeting ion channels is known to be notoriously difficult. Assaying ion channel activity with patchclamp recording of ion channel currents has very low screening throughput, although this problem is now largely alleviated by recent advances in cell line-based fluorescent assays and automated electrophysiology $y^{2,3}$. However, existing in vitro assays using cell lines expressing a particular ion channel could not overcome the problem that the channel in their native tissues exists in a cellular and physiological environment substantially different from that in the cell line, hence exhibits different molecular and functional properties. For example, accessory subunits usually dramatically alter the pharmacological properties of ion channels ${ }^{4,5}$. An efficient screening tool thus should examine the function of ion channels in the physiological context.

Most types of ion channels are found in C. elegans and are known to regulate worm behaviors ${ }^{6-10}$. Like their human homologs, C. elegans channels usually consist of pore-forming and accessory subunits ${ }^{10,11}$. Many mechanisms underlying the biogenesis of ion channels are conserved from C. elegans to humans ${ }^{1-13}$. Based on these findings and cumulative knowledge regarding ion channel mutations associated with human diseases, we hypothesized that expression of disease-relevant human ion channels in C. elegans would disturb normal electrical signaling and hence cause phenotypic defects in the transgenic worms. Compounds that modulate human ion channel function could be identified by examining their effects on the phenotypes of the transgenic worms. Phenotype-based screening in C. elegans has been used in dissecting mechanism of drug action and screening for new drugs ${ }^{14-16}$. In this study, we specifically targeted ion channel function using phenotype-based screening in C. elegans models of channelopathies, and demonstrated that specific smallmolecule modulators could be identified for correcting the defective function of mutant ion channels associated with channelopathies, e.g., Long-QT syndrome (LQTS).

Human ether-a-go-go-related gene ${ }^{17}$ (hERG) encodes a voltagegated $\mathrm{K}^{+}$channel that mediates cardiac $I_{\mathrm{Kr}}$ currents. Dysfunction of hERG $\mathrm{K}^{+}$channels due to genetic mutations or drug-induced inhibition results in LQTS ${ }^{18-22}$, a life-threatening cardiac arrhythmia that affects 1 in 2500 live births and results in about 5000 death each year in US alone ${ }^{23}$. Due to the fatal side effects of causing LQTS, several drugs have been withdrawn from the market since 1990s and the FDA now requires to test the drug effect on hERG activity during preclinical safety evaluation ${ }^{22,24}$. Most of hERG mutations underlying type 2 LQTS (LQTS2) suppress $I_{\mathrm{Kr}}$ currents by causing trafficking defects ${ }^{25,26}$. The mutant hERG $\mathrm{K}^{+}$channels are usually able to elicit normal or near normal $I_{\mathrm{Kr}}$ currents if they are properly transported to the plasma membrane by experimental manipulations, e.g., lowering the incubation temperature ${ }^{27,28}$. Pharmacological correction of the trafficking defect thus represents a new promising strategy for treating LQTS.

In this study, we generate channelopathy animal models by expressing chimeric hERG or trafficking-defective hERG mutant channels in C. elegans. Phenotype-based screens in the transgenic worms identify alphitolic acid (ALA) as a novel hERG trafficking inhibitor and two protein kinase C (PKC) activators, Prostratin and ingenol-3,20-dibenzoate (IDB), as functional correctors of some LQTS2 mutant channels. Thus, we provide an in vivo system for the discovery of ion channel modulators.

\section{Results}

Generation of C. elegans animal models of channelopathies. Human ion channels share high similarity with their C. elegans homologs, especially in their transmembrane domains ${ }^{29}$. To overcome the possible barrier of expressing human ion channels in C. elegans, we first made human-worm chimeric ion channels according to the strategy described in Fig. 1a. Ion channel inhibitors and activators often target at transmembrane domains and/ or loops at the membrane periphery of the channels. We thus assumed that a chimeric ion channel consisting of transmembrane domains and their connecting loops of a human ion channel, and N-/C-terminus of its C. elegans homolog, would largely maintain pharmacological properties of the human ion channel and could be functionally expressed in C. elegans. A gainof-function mutation was introduced into the channel (named as chimeric ion channel 1, Fig. 1a) to induce clear behavioral defects in the transgenic worms expressing the channel, and drugs that alleviate the phenotypic defects could be inhibitors of the channel (Fig. 1b). For screening channel activators or functional correctors of a mutant channel, a second mutation, which is a loss-offunction mutation, was introduced into the channel (named as chimeric ion channel 2, Fig. 1a), and transgenic worms expressing this channel should show no or weak behavioral defects due to the reduced channel function. Small molecules that aggravate the worms' behavioral defects are potential activators of the channel (Fig. 1b).

According to the above strategy, we made C. elegans models of channelopathies for identifying chemical modulators of hERG $\mathrm{K}^{+}$ channels. The hERG $\mathrm{K}^{+}$channel shows homology with the $C$. elegans ERG-type $\mathrm{K}^{+}$channel UNC-103 (Supplementary Fig. 1a), which is widely expressed in C. elegans head neurons and vulva muscles and regulates worms' locomotion, egg-laying, and male mating behaviors ${ }^{6,7}$. We first tried to express full-length hERG $\mathrm{K}^{+}$channels in C. elegans, but failed due to mistrafficking of the channel. We then constructed a GFP fused chimeric hERG

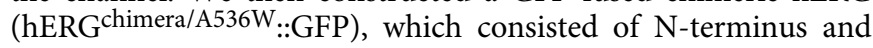
C-terminus of UNC-103, transmembrane, and cyclic nucleotidebinding domains of hERG, and a gain-of-function mutation $\mathrm{A} 536 \mathrm{~W}^{30}$ in the S4 segment of the hERG $\mathrm{K}^{+}$channel (Supplementary Fig. 1b). The mutation A536W, which switches the voltage-dependent activation of hERG $\mathrm{K}^{+}$channels to a much more negative potential ${ }^{30}$, was expected to alter cell excitability and consequently induce behavioral defects in worms. Indeed, expression of unc-103 promoter-driven hERG ${ }^{\text {chimera/A536W:: }}$ GFP proteins in N2 wild-type worms generated transgenic worms ( $h E R G^{\text {chimera/A536W }}$ ) with clear defects in locomotion and egg-laying (Fig. 1c-e). Fluorescent images suggested that hERG chimera/A536W::GFP channels could be delivered to the plasma membrane (Fig. 1f). In addition, expression of hERG ${ }^{\text {chi- }}$ mera/A536W rescued the copulatory spicule protraction defect caused by a loss-of-function mutation in the unc-103 gene in male worms (Supplementary Fig. 1c). Taken together, these data suggest that hERG ${ }^{\text {chimera/A536W }}$ could affect worms' behaviors.

Next, we examined whether behavioral defects in worm models of channelopathies could be rescued by known specific blockers of these channels. Cells of $C$. elegans are relatively inaccessible for most chemical compounds because of their cuticles and xenobiotic efflux pumps in the intestine. To facilitate penetration of compounds into the worm, we generated acs-20; $h E R G^{\text {chimera/A536W mutant worms }}$

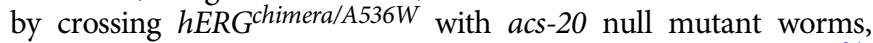
whose cuticles have increased permeability for small molecules ${ }^{31}$. To further enhance the targeting of the compounds, we fed these worms with bacteria expressing double-strand RNAs targeting ifd-2 and c15c7.5, two genes encoding nonessential intestinal intermediate filament proteins that impede the effects of ingested compounds ${ }^{32}$. We found that the phenotypes in the 
a

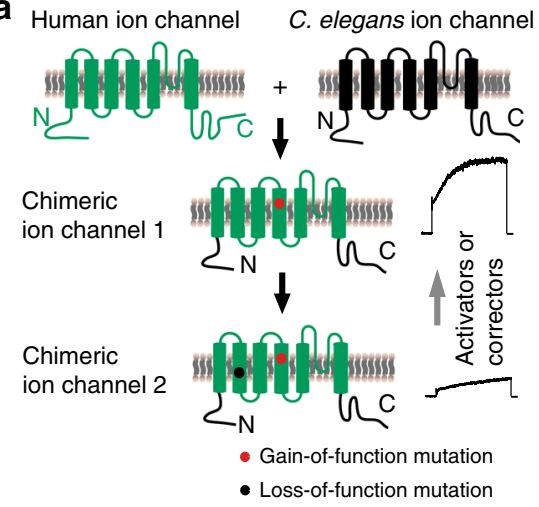

b

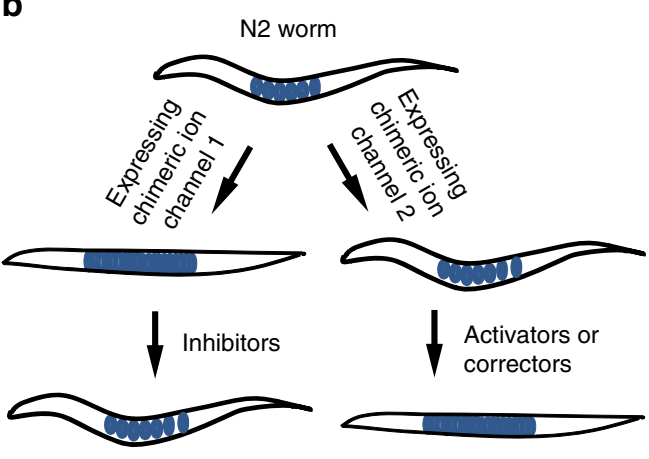

C

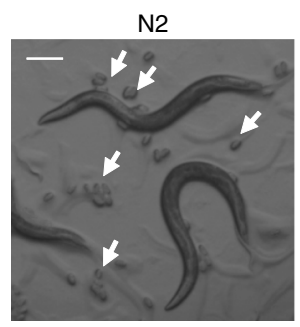

d

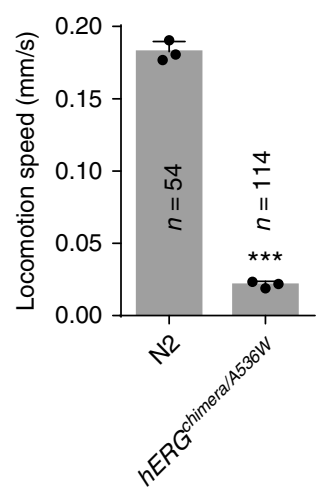

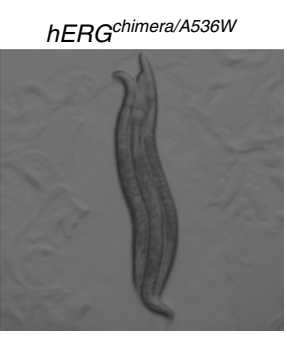

e

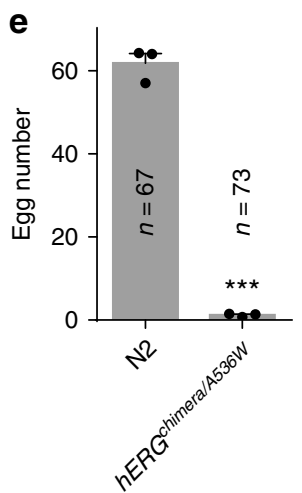

f
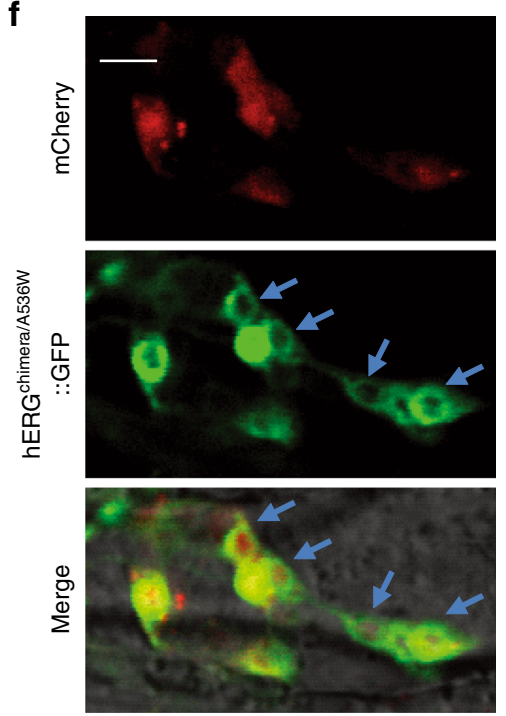

Fig. $1 \mathrm{C}$. elegans models of channelopathies show behavioral defects. a Schematic illumination of chimeric ion channels. The chimeric ion channels consist of transmembrane domains and their connecting loops of a human ion channel, and N-/C-terminus of its $\mathrm{C}$. elegans homolog. The gain-of-function mutation was expected to alter cell excitability and consequently induce behavioral defects in transgenic worms. b Schematic illumination of generating animal models of channelopathies and strategy for drug screenings. Transgenic worms expressing chimeric ion channel 1 proteins will exhibit clear behavior defects. Drugs that alleviate the phenotypic defects could be inhibitors of the channel. Transgenic worms expressing chimeric ion channel 2 proteins will show no or weak behavior defects due to the reduced channel function, and drugs aggravate the worms' behavioral defects are potential activators or functional correctors of the channel. c Microscopy images of wild-type N2 and $h E R G$ Chimera/A536W transgenic worms. Scale bar, $200 \mu M$. White arrows indicate C. elegans eggs. d, e Locomotive (d) and egg-laying (e) behaviors of of young adult wild-type N2 and $h E R G C h i m e r a / A 536 W$ transgenic worms. f Fluorescent images of worms expressing both mCherry and hERGchimera/A536W.:GFP proteins that were driven by the unc-103 promoter. Blue arrows indicate head neurons. Scale bar, $10 \mu \mathrm{m}$. All data shown are mean \pm s.e.m. ${ }^{\star \star \star} P<0.001$ (Student's $t$-tests for d, e)

$h E R G^{\text {chimera } / A 536 W}$ worms were ameliorated by individually adding $10 \mu \mathrm{M}$ well-known hERG blockers, and these effects were markedly enhanced when the acs-20 null mutation was introduced in the transgenic worms (Supplementary Fig. 2). This result indicates that drugs could be efficiently delivered to their targets, and modified human ion channels expressed in the transgenic worms are functional and the cause of behavioral defects.

A small-molecule screen identifies a new hERG trafficking inhibitor. Next, we examined whether acs-20; $h E R G^{\text {chimera/A536W }}$ worms could be used for identification of hERG $\mathrm{K}^{+}$channel modulators. To identify unknown hERG inhibitors, we screened about 4000 small molecules for their suppression of behavioral defects in acs-20; $h E R G^{\text {chimera/A536W }}$ worms (Fig. 2a). Dozens of compounds were found to alleviate the phenotypic defects to some extents. Among them, alphitolic acid (ALA) clearly restored locomotion and egg-laying behaviors of the tested worms (Fig. 2b). Additional test using HEK293T cells expressing wildtype hERG $\mathrm{K}^{+}$channels showed that acute treatment with either 1 or $10 \mu \mathrm{M}$ ALA did not change the current density (Fig. 2c), suggesting that ALA did not act directly on hERG $\mathrm{K}^{+}$channel permeation. By contrast, long-term (24-32 h) treatment with ALA led to reduced current density of this channel in a dosedependent manner. The median inhibition concentration $\left(\mathrm{IC}_{50}\right)$ was $8.2 \mu \mathrm{M}$, as determined by analyzing changes in deactivating tail currents at the membrane potential of $-140 \mathrm{mV}$ (Fig. 2d-f). Western blotting data showed that hERG proteins appeared as two bands corresponding to the endoplasmic reticulum (ER)- 
a

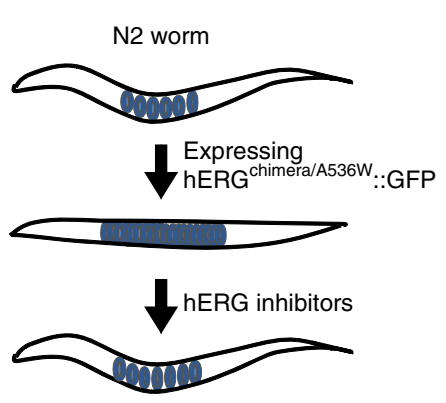

b

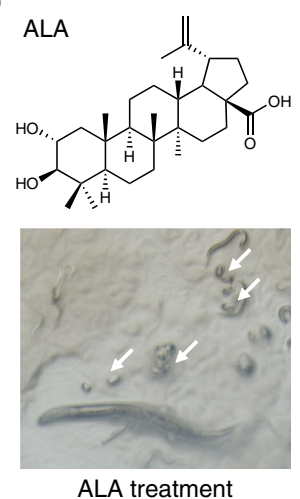

C ALA acute treatment

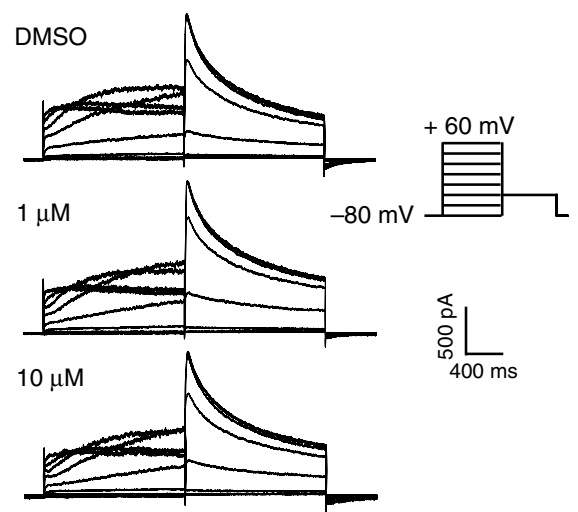

e

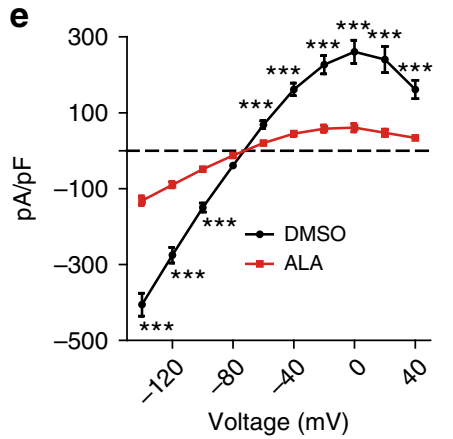

d

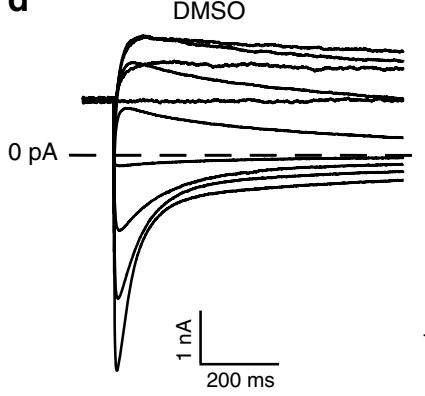

$\mathbf{f}$

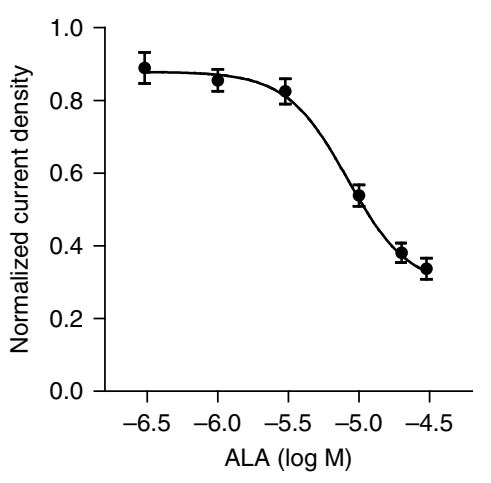

ALA treatment $24 \mathrm{~h}$

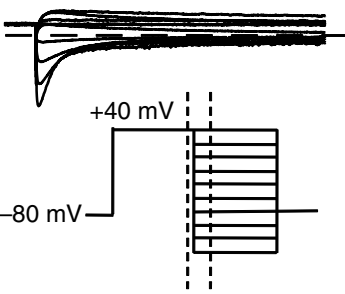

g
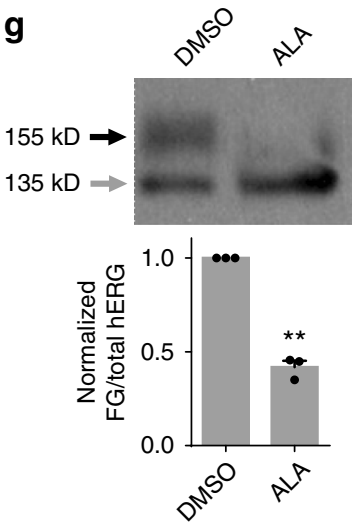

h

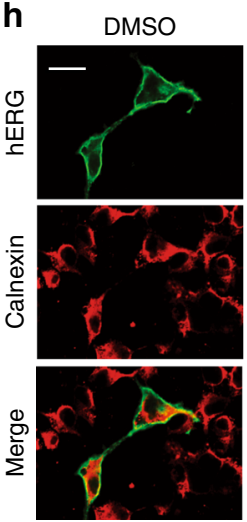

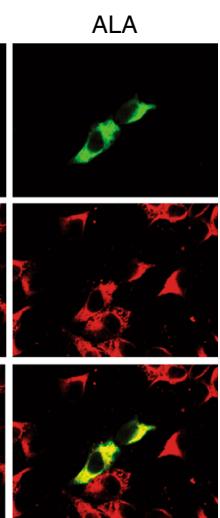

Fig. 2 A small-molecule screen in C. elegans identifies a novel hERG trafficking inhibitor. a Schematic illumination of the screening assay. $\mathbf{b}$ Molecular formula of alphitolic acid (ALA, upper) and microscopic images of acs-20;hERGchimera/A536W transgenic worms in the presence of $20 \mu \mathrm{M}$ ALA in cultivating plates (lower). White arrows indicate eggs. c Representative whole-cell currents of HEK293T cells expressing wild-type hERG channels before or after treated with 1 or $10 \mu \mathrm{M}$ ALA. $n=6$ cells. d, e Representative whole-cell currents and I/V curves of wild-type hERG channels expressed in HEK293T cells treated with DMSO $(n=19)$ or $20 \mu \mathrm{M}$ ALA $(n=25)$ for $24 \mathrm{~h}$. The protocol is shown in the lower right of $\mathbf{d}$, and traces at the time course between two dashed lines are shown. $\mathbf{f}$ Dose-dependent effects of long-term ALA treatment on the current densities of wild-type hERG channels recorded at a membrane potential of $-140 \mathrm{mV} . n \geq 20$ cells per dose were recorded and analyzed. $\mathbf{g}$ Western blot analysis of hERG proteins expressed in HEK293T cells treated with DMSO or $20 \mu \mathrm{M}$ ALA for $24 \mathrm{~h}$ (top), and quantitantive analysis of the ratio of fully glycosylated (FG, $155 \mathrm{kD}$ ) to total hERG proteins (down). Black and gray arrows indicate $155 \mathrm{kD}$ and $135 \mathrm{kD}$ bands of hERG proteins, respectively. $\mathbf{h}$ Immunostaining of hERG and ER marker protein Calnexin in HEK293T cells treated with DMSO or $20 \mu \mathrm{M}$ ALA for $24 \mathrm{~h}$. Scale bar: $20 \mu \mathrm{m}$. Data shown are mean \pm s.e.m. ${ }^{\star \star} P<0.01$, ${ }^{\star \star \star} P<0.001$ (Student's $t$-tests for e, $\mathbf{g}$. All experiments were performed at least three times

resident (with core glycosylation, $\sim 135 \mathrm{kD}$ ) and post-ER (with fully glycosylation, $\sim 155 \mathrm{kD}$ ) forms (Fig. $2 \mathrm{~g}$ ). Long-term treatment with ALA reduced the hERG protein levels of the $155 \mathrm{kD}$, but not the $135 \mathrm{kD}$ band (Fig. $2 \mathrm{~g}$ ). Furthermore, immunostaining of HEK293T cells expressing hERG $\mathrm{K}^{+}$channels also showed that ALA inhibited protein trafficking of hERG $\mathrm{K}^{+}$channels (Fig. $2 \mathrm{~h}$ ). These results suggest that ALA is a novel hERG protein trafficking inhibitor. Together, screening assays in worms expressing hERG $\mathrm{K}^{+}$channels could identify new hERG inhibitors.
Identification of functional correctors of LQTS2 mutant channels. The majority of LQTS2-related mutations suppress the $I_{\mathrm{Kr}}$ currents by retaining hERG $\mathrm{K}^{+}$channels in the $\mathrm{ER}^{25,26}$. Pharmacological correction of trafficking defects thus represents a promising therapeutic strategy for LQTS ${ }^{27,28,33,34}$. In order to generate an animal model of LQTS, a trafficking-defective LQTS2 mutation A561V 27,35 was introduced to the hERG ${ }^{\text {chimera/ }}$ A536W protein (Fig. 3a). As expected, the transgenic worms hERG ${ }^{\text {chimera/A536W/A561V }}$ (expressing hERG ${ }^{\text {chimera/A536W/A561V:: }}$ 
a

hERG ${ }^{\text {chimera/A536W/A561V }}$ channel

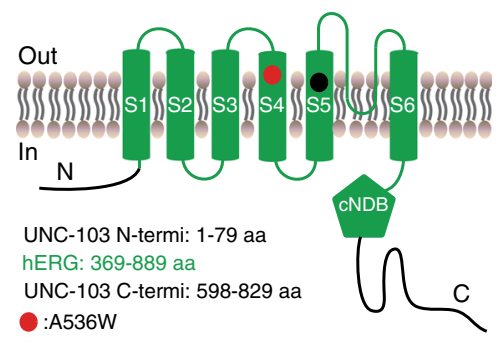

:A536W

-:A561V b

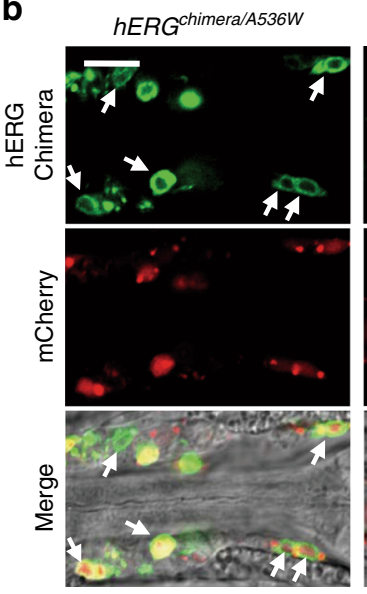

C

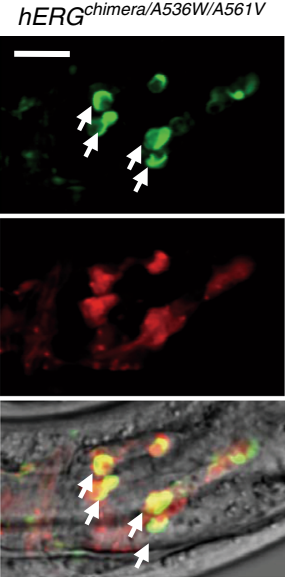

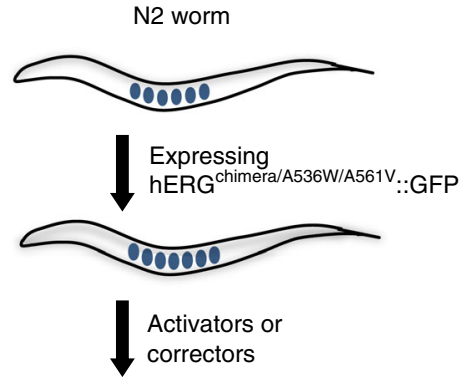

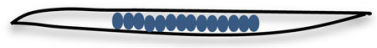

Selection according to

phenotypes and GFP reporter d

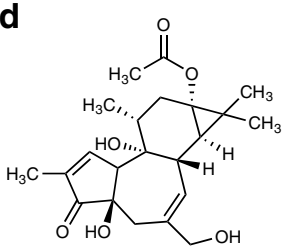

Prostratin

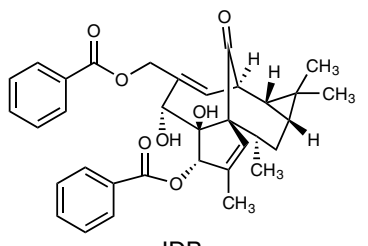

IDB

e

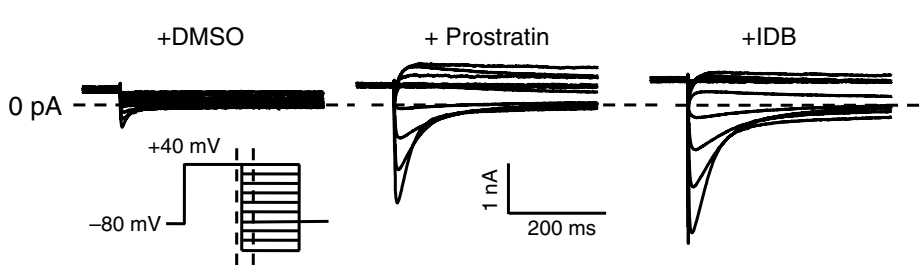

f

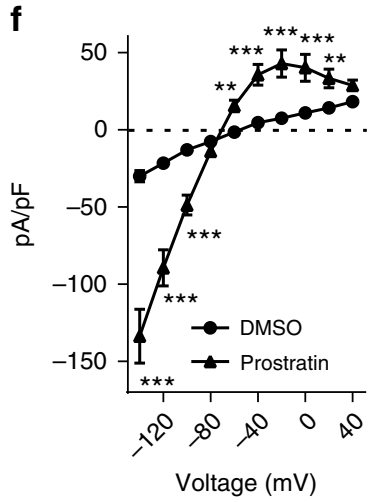

h

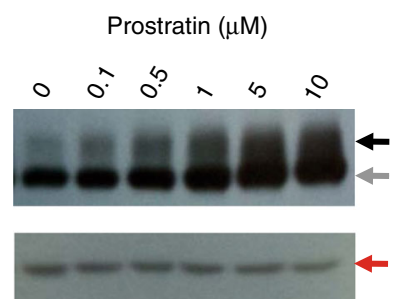

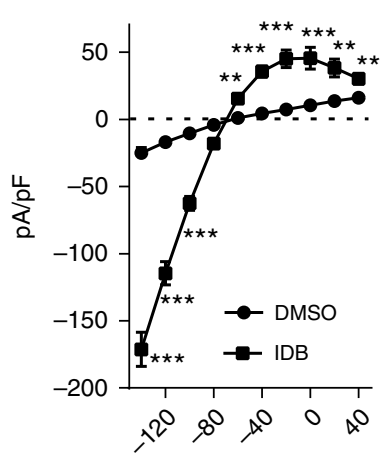

Voltage $(\mathrm{mV})$

IDB ( $\mu \mathrm{M})$

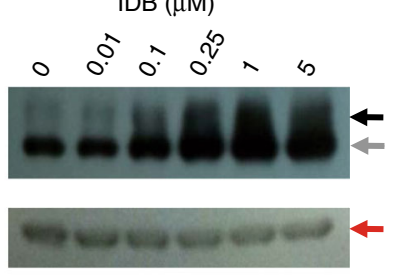

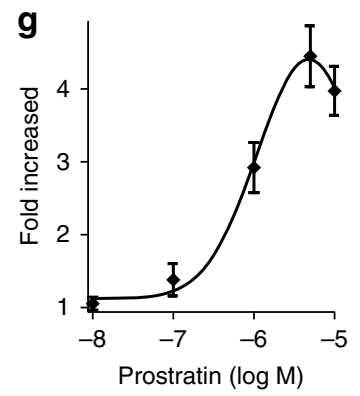

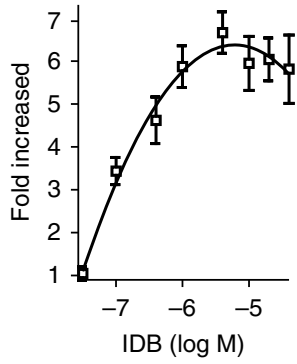

i

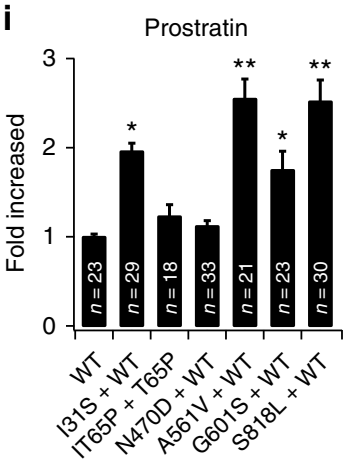

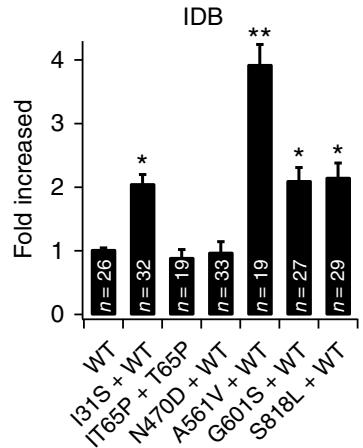

Fig. 3 Small-molecule screens identify functional correctors of hERGA561V. a Schematic illumination of construction of hERGchimera/A536W/A561V . Mutations A536W and A561V are indicated by a red and a black circle, respectively. aa amino acids. b Localization of hERGchimera/A536W::GFP and hERGchimera/A536W/A561V::GFP expressed in C. elegans neurons. White arrows indicate head neurons. Scale bar, $10 \mu \mathrm{m}$. c Schematic illumination of screening strategy for the discovery of functional correctors. Briefly, we first searched for compounds that caused locomotive and egg-laying defects, and then the candidate compounds were further selected according to surface expression of hERGchimera/A536W/A561V::GFP proteins. $\mathbf{d}$ Molecular formulas of Prostratin and IDB. e, f Representative whole-cell currents (e) and I/V curves (f) of hERGWT-hERGA561V channels expressed in HEK293T cells treated with DMSO $(n=26), 3 \mu \mathrm{M}$ Prostratin $(n=21)$ or $2 \mu \mathrm{M}$ IDB $(n=25)$. $\mathbf{g}$ Dose-dependent effects of Prostratin and IDB on the current density of hERGWT. hERGA561V channels recorded at a membrane potential of $-140 \mathrm{mV}$. About 20 cells per dose were recorded and analyzed. $\boldsymbol{h}$ Effects of Prostratin and IDB on the protein trafficking of hERGWT-hERGA561V channels. Black, gray, and red arrows indicate $155 \mathrm{kD}, 135 \mathrm{kD}$ bands of hERG proteins, and tubulin, respectively. i Effects of Prostratin and IDB on the current densities of LQTS-related hERG mutant channels. The ratio of hERGWT/hERGA561V was 2:1 for $\mathbf{e}, \mathbf{f}, \mathbf{g}$, and 1:1 for $\mathbf{h}, \mathbf{l}$. The ratio of hERGWT/other hERG mutant was 1:1. All experiments were performed at least three times. Data shown are mean $\pm \mathrm{s}$.e.m. ${ }^{\star} P<0.05,{ }^{\star \star} P<0.01,{ }^{\star \star \star} P<0.001$ (Student's $t$-tests for $\mathbf{f}$; one-way ANOVA Dunnett's test for $\mathbf{i}$ ) 
GFP proteins) showed GFP reporters in the neuronal cytoplasm (Fig. 3b), indicating mistrafficking of these channel proteins. Consistently, hERG chimera/A536W/A561V worms exhibited nearly normal behaviors (Supplementary Movies 1-3).

The hERG ${ }^{A 561 V} \mathrm{~K}^{+}$channels could be functional if they reach to the plasma membrane ${ }^{27}$. We hypothesized that if a compound promotes the surface expression of hERG ${ }^{\text {chimera/A536W/A561V:: }}$ GFP, the transgenic worms would show locomotion and egglaying defects. We thus generated acs-20; $h E R G^{\text {chimera/A536W/A56IV }}$ worms by crossing acs-20 null mutant worms with $h E R G^{\text {chimeral }}$ $A 536 \mathrm{~W} / \mathrm{A5} 61 \mathrm{~V}$, and screened for novel functional correctors of hERG $^{\text {chimera/A536W/A561V }}$ according to the strategy depicted in the Fig. 3c. Among 10,600 compounds screened at a final concentration of $20 \mu \mathrm{M}$, we identified six hits, among which two compounds Prostratin and IDB (Fig. 3d and Supplementary Fig. 3) were known as PKC activators.

The A561V mutation is known to cause strong dominantnegative suppression of wild-type hERG (hERGWT) protein trafficking, resulting in reduced channel function at the surface ${ }^{35,36}$. Strikingly, either Prostratin or IDB treatment enhanced the current density of hERG $\mathrm{K}^{+}$channels in a dose-dependent pattern when we co-expressed hERGWT and hERG ${ }^{\mathrm{A} 561 \mathrm{~V}}$ at a ratio of 2:1 (Fig. 3e-g). The half maximal effective concentrations (EC50) of Prostratin and IDB were $0.95 \mu \mathrm{M}$ and $0.042 \mu \mathrm{M}$, respectively (Fig. 3g). We then examined whether these compounds affect protein trafficking of hERG mutant channels. In this assay, we co-expressed hERG ${ }^{\mathrm{WT}}$ and mutated hERG $\mathrm{K}^{+}$ channels at a ratio of 1:1 because hERG mutations are heterozygous in most LQTS2 patients. Western blotting experiments showed that both Prostratin and IDB promoted trafficking of hERG ${ }^{\text {WT }}$-hERG ${ }^{\mathrm{A} 561 \mathrm{~V}}$ channels in a dose-dependent pattern, as reflected by the elevated $155 \mathrm{kD}$ hERG proteins (Fig. $3 \mathrm{~h}$ and Supplementary Fig. 4), which distributed outside the ER. In addition to the A561 site, hERG $\mathrm{K}^{+}$channels with other LQTSrelated mutations such as I31S, G601S, and S818L were found to exhibit enhanced function after treatment with $3 \mu \mathrm{M}$ Prostratin or $2 \mu \mathrm{M}$ IDB, while the channels with T65P, N470D were not affected by the drug administration (Fig. 3i). Moreover, neither of these two compounds influenced the function and protein trafficking of hERGWT channels (Supplementary Fig. 5). Together, Prostratin and IDB specifically promote the function of some trafficking-defective hERG mutant channels.

Prostratin and IDB restore the function of LQTS2 mutant channels through PKC $\varepsilon$ signaling. Both Prostratin and IDB are $\mathrm{PKC}$ activators. Prostratin is non-selective, and IDB is specific for the PKCe. We then examined whether the rescue of trafficking defects by Prostratin and IDB results from activation of PKC $\varepsilon$. We found that downregulation of PKCe, but not PKC $\beta$, abolished the effect of IDB and Prostratin on the current density (Fig. $4 \mathrm{a}-\mathrm{c}$ and Supplementary Fig. 6) and protein trafficking of hERGWT-hERGA561V channels (Fig. 4d). Furthermore, overexpression of $\mathrm{PKC \varepsilon}$ enhanced current densities and promoted protein trafficking of $\mathrm{hERG}^{\mathrm{WT}}$-hERG ${ }^{\mathrm{A} 561 \mathrm{~V}}$ channels (Fig. 4e, f). Together, these results demonstrate that Prostratin and IDB recover trafficking of hERG mutant channels through the PKCe signaling.

Previous studies ${ }^{37}$ have shown that hERG contains 18 putative PKC-dependent phosphorylation sites (Fig. 5a). To explore whether the action of Prostratin and IDB depends on phosphorylation of hERG mutant $\mathrm{K}^{+}$channels, 17 out of 18 PKC phosphorylation sites (except the T74 site, which prevents functional expression of hERG $\mathrm{K}^{+}$channel $^{37}$ ) of the channel were mutated to alanine to block the phosphorylation of these sites. We found that these mutations largely abolished the effects of IDB and Prostratin on correcting the functional defects in LQTS2 mutant channels (Fig. 5b). To further explore which phosphorylation site is required for the function of IDB and Prostratin, we generated three mutated hERG $\mathrm{K}^{+}$channels with a lack of PKC phosphorylation sites in the N-terminus, the transmembrane domains, or the C-terminus. We found that only blockage of phosphorylation sites in the transmembrane domains diminished the function of IDB and Prostratin (Fig. 5c-e). Further studies showed that a phosphorylation site of hERG $\mathrm{K}^{+}$channels at $\mathrm{S} 606$ (Fig. 5f), but not S636 and T670 (Supplementary Fig. 7), was required for the function of IDB and Prostratin. We next mutated S606 to E606 to mimic the phosphorylation of hERG $\mathrm{K}^{+}$ channels, and found that the mutation S606E in hERGA561V markedly increased the current density of hERG ${ }^{\mathrm{WT}}$-hERG ${ }^{\mathrm{A} 561 \mathrm{~V}}$ (Fig. 5g), suggesting that the phosphorylation of this site promotes the function of the LQTS2 mutant channel. Taken together, our results suggest that IDB and Prostratin activate the PKCe signaling and consequently phosphorylate S606 at the pore region of the channel to correct the defective function of LQTS2 mutant channels.

Identified compounds restore electrophysiology of cardiomyocytes carrying a LQTS2 mutation. The hERG $\mathrm{K}^{+}$channel plays a major regulatory role in the re-polarization phase of cardiac action potentials. Human-induced pluripotent stem cellderived cardiomyocytes (hiPSC-CMs) from LQTS2 patients (with a heterozygous A561V mutation in hERG) showed diminished $I_{\mathrm{Kr}}$ currents and prolonged re-polarization durations ${ }^{27}$. We then generated a heterozygous A561V mutation in hiPSCs by CRISPR/ Cas9-mediated genome editing (Fig. 6a and Supplementary Fig. 8a), and differentiated these hiPSCs into cardiomyocytes

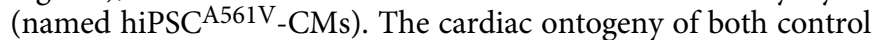
hiPSC-CMs and hiPSC ${ }^{\mathrm{A} 561 \mathrm{~V}}-\mathrm{CMs}$ was confirmed by analyses of cardiac cell-specific transcriptional, structural, and functional markers (Fig. 6b, c).

We found that endogenous $I_{\mathrm{Kr}}$ currents, isolated by the hERG blocker E-4031, in hiPSC ${ }^{\mathrm{A} 561 \mathrm{~V}_{-} \mathrm{CMs}}$ were significantly decreased in comparison with those in control hiPSC-CMs (Fig. 6d, e). In consistence with this result, immunostaining of hiPSC ${ }^{\mathrm{A} 561 \mathrm{~V}_{-} \mathrm{CMs}}$ showed that hERG ${ }^{\mathrm{A} 561 \mathrm{~V}}$ proteins accumulated in the cytoplasm (Supplementary Fig. 8b). Concurrently, the $\mathrm{APD}_{90}$ (action potential duration at $90 \%$ of full re-polarization) of atrial-like

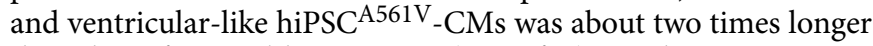
than that of control hiPSC-CMs (Fig. 6f, g). Furthermore, action potentials in 5 out of 22 hiPSC $^{\mathrm{A} 561 \mathrm{~V}}$-CMs exhibited early afterdepolarization (EAD), the harbinger of cardiac arrhythmias in LQTS, while none of action potentials in control hiPSC-CMs $(n=25)$ showed EADs (Supplementary Fig. 8c, d). Thus, the electrophysiological phenotypes in hiPSC ${ }^{\mathrm{A} 561 \mathrm{~V}_{-} \mathrm{CMs} \text { were similar }}$ to those in LQTS2 patient-specific hiPSC-CMs ${ }^{27,38}$.

We then explored whether Prostratin and IDB could correct the electrophysiological defects of hiPSC ${ }^{\mathrm{A} 561 \mathrm{~V}_{-} \mathrm{CMs} \text {.We found }}$ that long-term $(48 \mathrm{~h})$ administration of Prostratin $(3 \mu \mathrm{M})$ or IDB $(2 \mu \mathrm{M})$ markedly enhanced the $I_{\mathrm{Kr}}$ currents in hiPSC ${ }^{\mathrm{A} 561 \mathrm{~V}_{-}} \mathrm{CMs}$ (Fig. 6d, e). Immunostaining results suggested that Prostratin and IDB ameliorated the trafficking defects of hERG ${ }^{A 561 V}$ proteins in

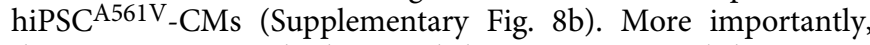
these two compounds shortened the action potential duration of hiPSC ${ }^{\mathrm{A} 561 \mathrm{~V}_{-} \mathrm{CMs}}$ to a level comparable with that of control hiPSC-CMs (Fig. 6f, g) and prevented the occurrence of EADs (Supplementary Fig. 8c, d). By contrast, these two compounds did not affect wild-type $I_{\mathrm{kr}}$ currents and the duration of action potentials in control hiPSC-CMs (Supplementary Fig. 9a-d). We further examined the effect of Prostratin and IDB on the transcription of hERG splice variants and other channels

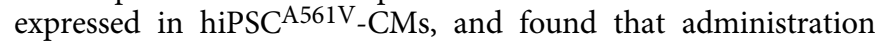


a

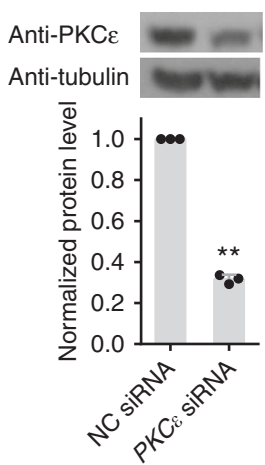

C

$$
\begin{aligned}
& \text { - NC siRNA; DMSO }(n=20) \\
& \text { — NC siRNA; Prostratin }(n=19) \\
& \text { — PKC } \varepsilon \text { siRNA; DMSO }(n=18)
\end{aligned}
$$$$
\text { - } P K C \varepsilon \text { siRNA; Prostratin }(n=17)
$$

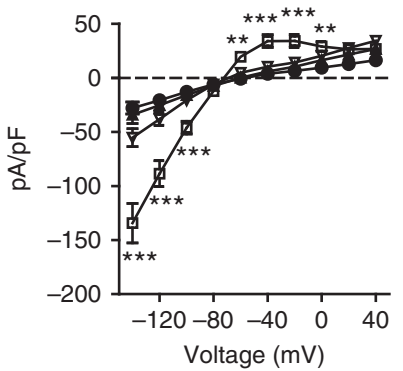

e

$$
\begin{aligned}
& \rightarrow \operatorname{Control}(n=28) \\
& \neq \operatorname{PKCE}(n=27)
\end{aligned}
$$

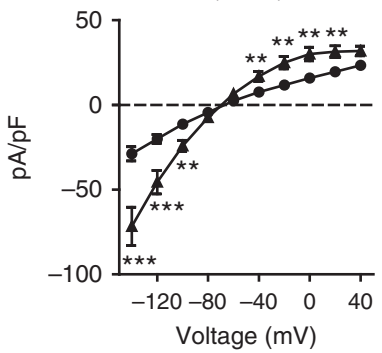

b

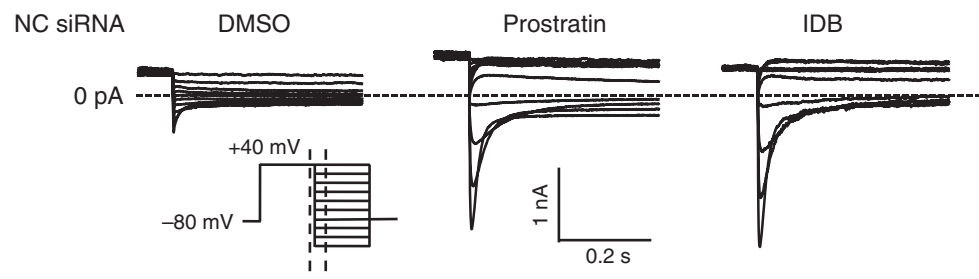

$$
\begin{aligned}
& P K C \varepsilon \text { siRNA DMSO } \\
& \rightarrow \mathrm{NC} \text { siRNA; DMSO }(n=18) \\
& \text { — NC siRNA; IDB }(n=17) \\
& \text { — PKC } \varepsilon \text { SiRNA; DMSO }(n=17)
\end{aligned}
$$

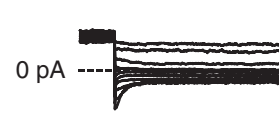

$\rightarrow P K C \varepsilon \operatorname{siRNA} ; \operatorname{IDB}(n=17)$

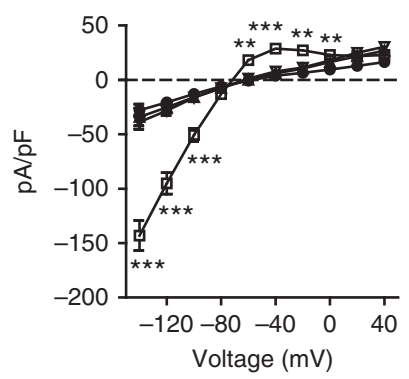

d

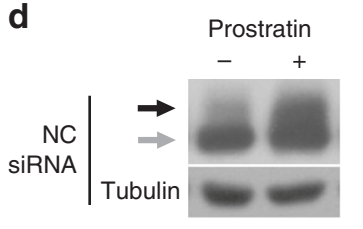

IDB
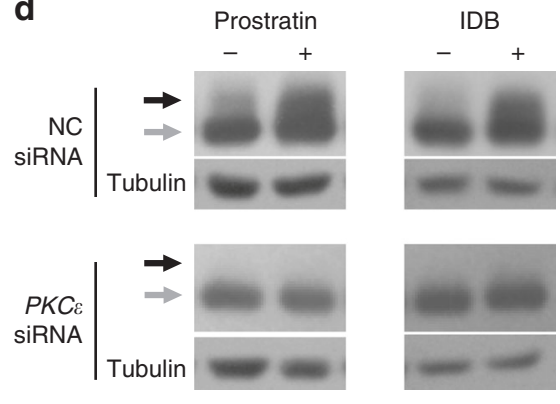

f

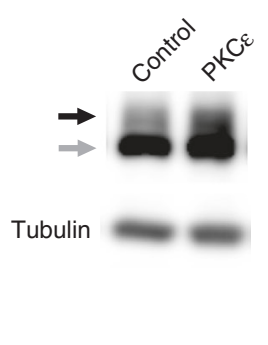

Fig. 4 The action of Prostratin and IDB requires activation of PKC $\varepsilon$ signaling. a The efficiency of siRNAs targeting $P K C \varepsilon$. A scrambled siRNA was used as a negative control (NC). b, c Effects of Prostrain and IDB on whole-cell currents (b) and current densities (c) of hERGWT-hERGA561V channels expressed in HEK293T cells treated with control or PKCE siRNAs. d Effects of Prostrain and IDB on protein trafficking of hERGWT-hERGA561V channels expressed in HEK293T cells treated with control or PKC $\varepsilon$ siRNAs. e, $\mathbf{f}$ Effects of PKC $\varepsilon$ over-expression on current densities (e) and protein trafficking (f) of hERGWT_ hERGA561V channels expressed in HEK293T cells. Black and gray arrows indicate $155 \mathrm{kD}$ and $135 \mathrm{kD}$ bands of hERG proteins, respectively. The ratio of hERGWT/hERGA561V was 2:1 for $\mathbf{b}, \mathbf{c}, \mathbf{e}$, and 1:1 for $\mathbf{d}, \mathbf{f}$. All experiments were performed at least three times. Data shown are mean \pm s.e.m. ${ }^{\star \star} P<0.01$, ${ }^{\star \star \star} P<0.001$ (one-way ANOVA Dunnett's test for $\mathbf{c}$, Student's $t$-tests for $\mathbf{a}, \mathbf{e}$ )

of Prostratin and IDB marginally influenced the expression of these examined ion channels (Supplementary Fig. 9e). Together, these data demonstrate that Prostratin and IDB correct electrophysiological abnormalities in LQTS2-specific hiPSC-CMs. Thus, small-molecule compounds similar to Prostratin and IDB are potential drugs for treating patients with LQTS2.

\section{Discussion}

The discovery of new ion channel drugs could be greatly facilitated by the availability of an economic, reliable, robust, and highthroughput in vivo assay. In this study, we generated C. elegans models of channelopathies by expressing disease-related hERG channels in the worms, and developed phenotype-based screening assays by which we successfully identified some novel channel modulators, illustrating the usefulness of this in vivo highthroughput screening method.

Theoretically, inherited mutations could result in dysfunctions of ion channels by the following mechanisms: abnormal transcription/translation, deficient assembly or protein trafficking, defective channel gating/kinetics, and altered channel permeability $^{22,33}$. Different disease-related mutant channels may need different strategies to rescue their function. For instance, the function of an ion channel mutant with abnormal transcription, translation, or defective trafficking may not be fully corrected by activators of ion channels. Current drug screenings, however, mainly focus on identification of compounds that modulate the gating and kinetics of ion channels. In this study, we have demonstrated that different screening methods could be designed by introducing specific disease-related channel mutations into $C$. 
a

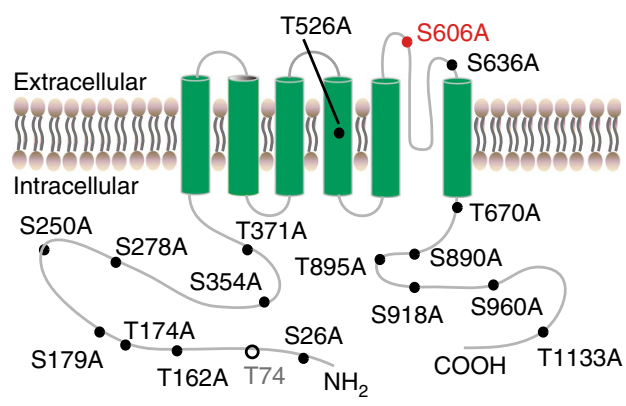

b - 17(A), 17(A)-A561V; IDB $(n=30)$

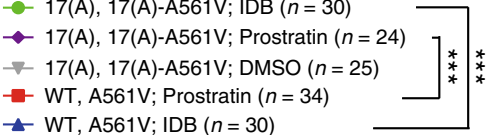

$\rightarrow \mathrm{WT}, \mathrm{A} 561 \mathrm{~V}$; DMSO $(n=25)$

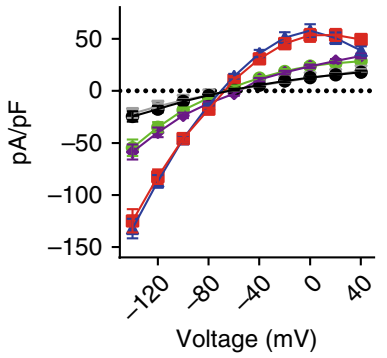

C

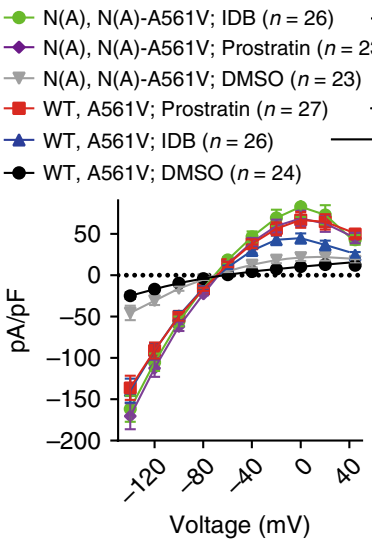

f

- S606A, S606A-A561V; IDB $(n=24)$

$\rightarrow$ S606A, S606A-A561V; Prostratin $(n=20)$

$\rightarrow$ S606A, S606A-A561V; DMSO $(n=18)$

- WT, A561V; Prostratin $(n=13)$

- WT, A561V; IDB $(n=14)$

$\rightarrow$ WT, A561V; DMSO $(n=21)$

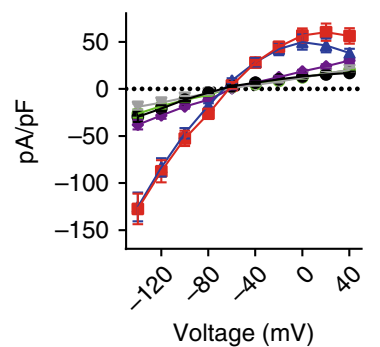

d

- T(A), T(A)-A561V; IDB $(n=25)$

$\rightarrow \mathrm{T}(\mathrm{A}), \mathrm{T}(\mathrm{A})$-A561V; Prostratin $(n=23)$

$\rightarrow \mathrm{T}(\mathrm{A}), \mathrm{T}(\mathrm{A})$-A561V; DMSO $(n=25)$

-WT, A561V; Prostratin $(n=25)$

$-\mathrm{WT}, \mathrm{A} 561 \mathrm{~V} ; \mathrm{IDB}(n=27)$

$\rightarrow$ WT, A561V; DMSO $(n=23)$

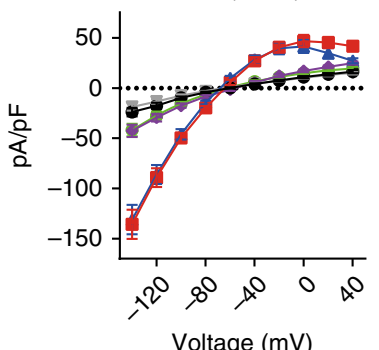

Voltage $(\mathrm{mV})$

g e

-C C(A), C(A)-A561V; IDB $(n=25)$

$\rightarrow \mathrm{C}(\mathrm{A}), \mathrm{C}(\mathrm{A})-\mathrm{A} 561 \mathrm{~V}$; Prostratin $(n=24)$

$\rightarrow$ C(A), C(A)-A561V; DMSO $(n=23)$

- WT, A561V; Prostratin $(n=23)$

- WT, A561V; IDB $(n=16)$

- WT, A561V; DMSO $(n=21)$

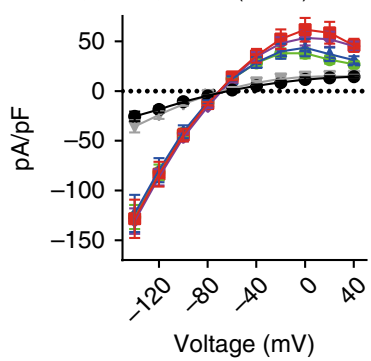

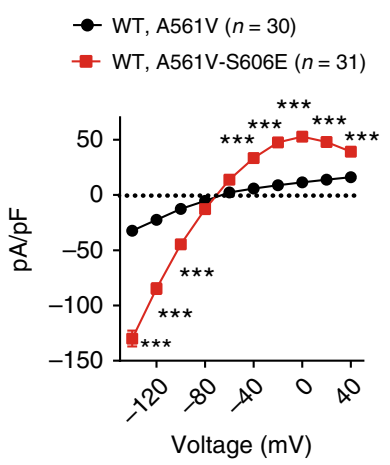

Fig. 5 The action of Prostratin and IDB depends on the phosphorylation of the S606 at the pore region of the hERG channel. a Schematic illumination of putative PKC phosphorylation sites and point mutations in hERG proteins. $\mathbf{b}$ Effects of Prostrain and IDB on the current densities of hERGWT-hERGA561V channels with a lack of 17 PKC phosphorylation sites (indicated as 17(A)). c-e Effects of Prostrain and IDB on the current densities of hERGWT-hERGA561V channels with a lack of PKC phosphorylation sites (S26A, T162A, T174A, S179A, S250A, S278A, S354A, and T371A) in the N-terminus (indicated as N (A)), PKC phosphorylation sites (T526A, S606A, S636A, and T670A) in the transmembrane domain (indicated as T(A)), or PKC phosphorylation sites (S890A, T895A, S918A, S960A, and T1133A) in the C-terminus (indicated as C(A)). f Effects of Prostrain and IDB on the current densities of hERGWT. hERGA561V channels with a lack of the S606 PKC phosphorylation site. $\mathbf{g}$ Effects of the mutation S606E on the current densities of hERGWT-hERGA561V channels. The ratio of hERGWT/hERGA561V was 2:1. All experiments were performed at least three times. Data shown are mean \pm s.e.m. ${ }^{\star \star \star} P<0.001$, n.s., not significant (one-way ANOVA Dunnett's test for $\mathbf{b}, \mathbf{c}, \mathbf{d}, \mathbf{e}$, and $\mathbf{f}$; Student's $t$ tests for $\mathbf{g}$ )

elegans disease models. Phenotype-base screens in these animal models could discover compounds that correct abnormal channel phenotypes caused by specific mutations.

The cumulative evidence suggests that defective protein biogenesis represents a rather common mechanism underlying many channelopathies, including LQTS, cystic fibrosis, episodic ataxia type 1, epilepsy, and hearing loss ${ }^{25,26,33,39-41}$. Despite extensive interests in pharmacological correction of defective protein biogenesis of ion channels, few such compounds have been found. A successful example is that a combination administration of $\mathrm{CFTR}^{\triangle \mathrm{F} 508}$ protein trafficking corrector VX-809 and CFTR chloride channel potentiator VX-770 was approved for the 
a

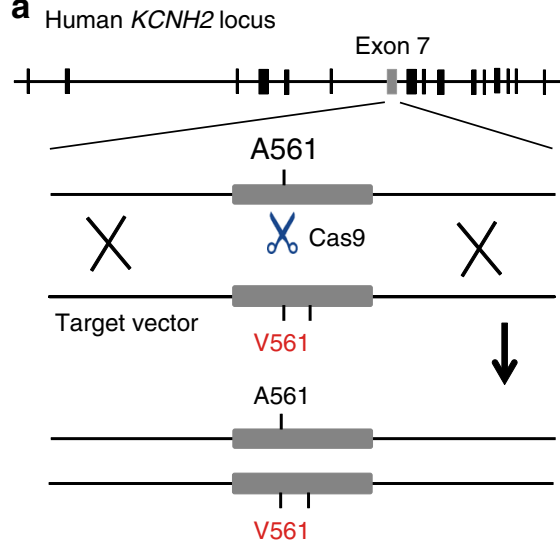

b

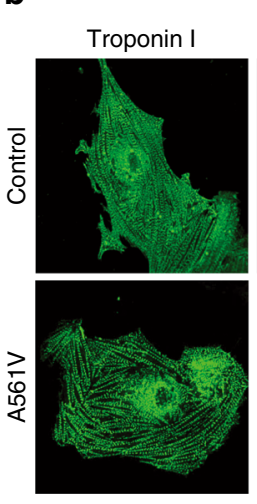

Sarcomeric

$\alpha$-actinin
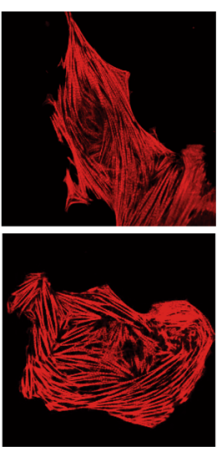

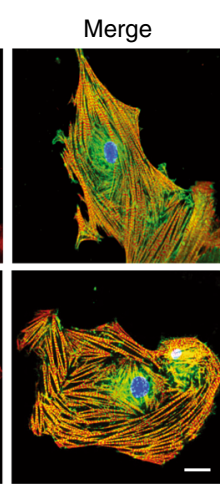

C

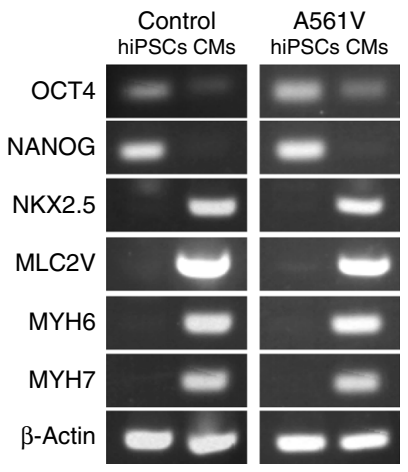

d Control

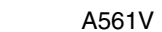

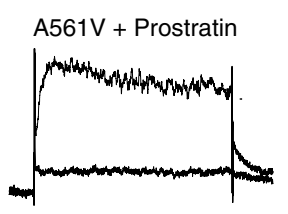

Baseline

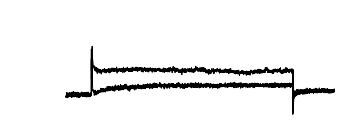

$+\mathrm{E} 4031$
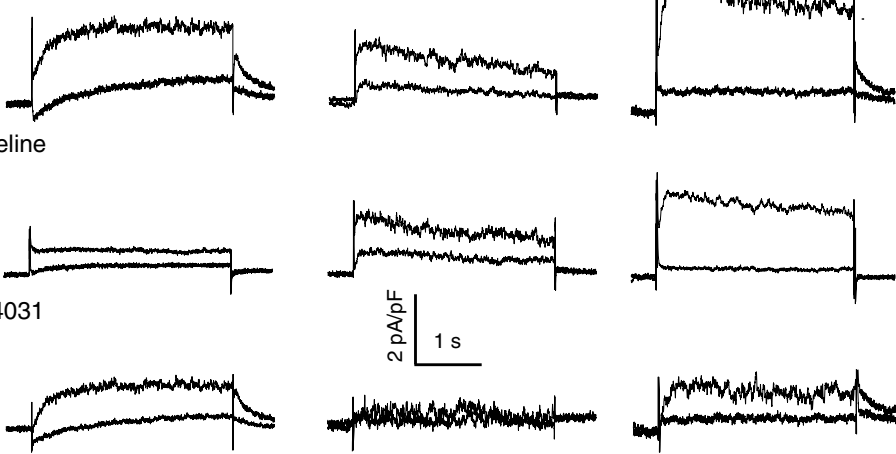

$I_{\mathrm{Kr}}=$ Baseline $-\mathrm{E} 4031$
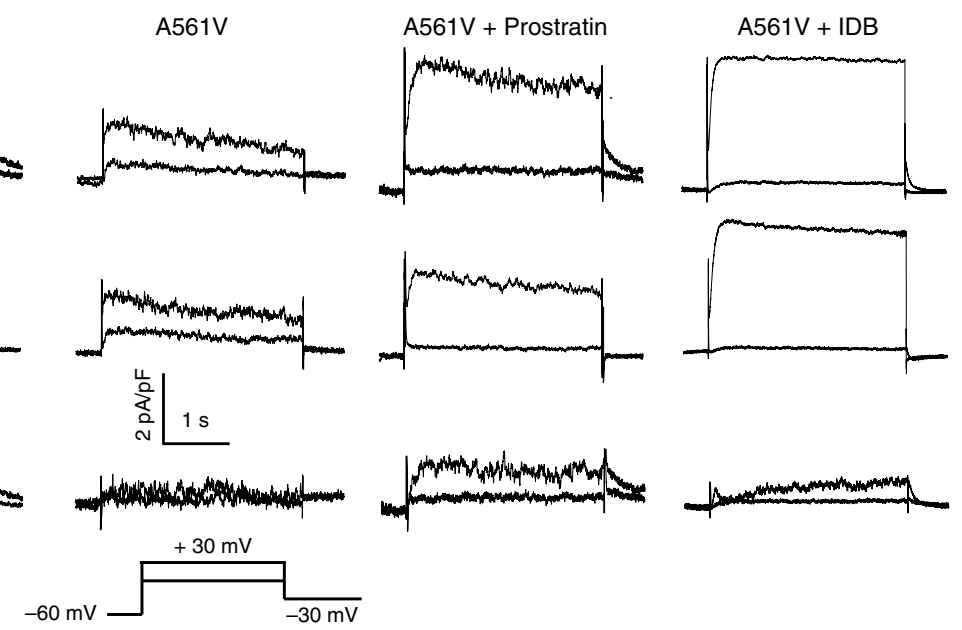

f

Atria-like
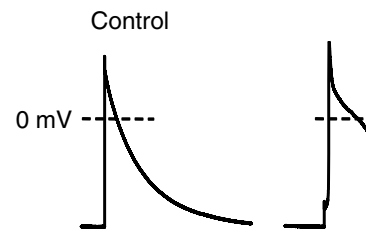

A561V
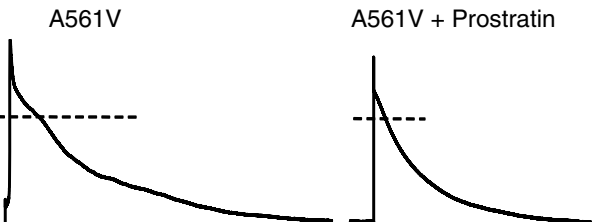

Ventricular-like
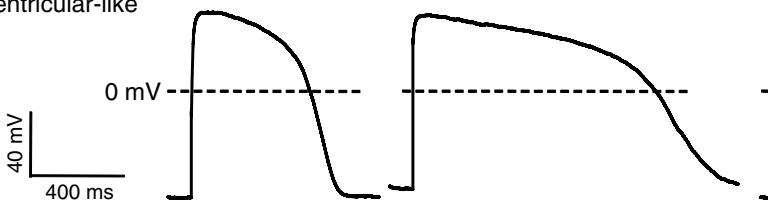
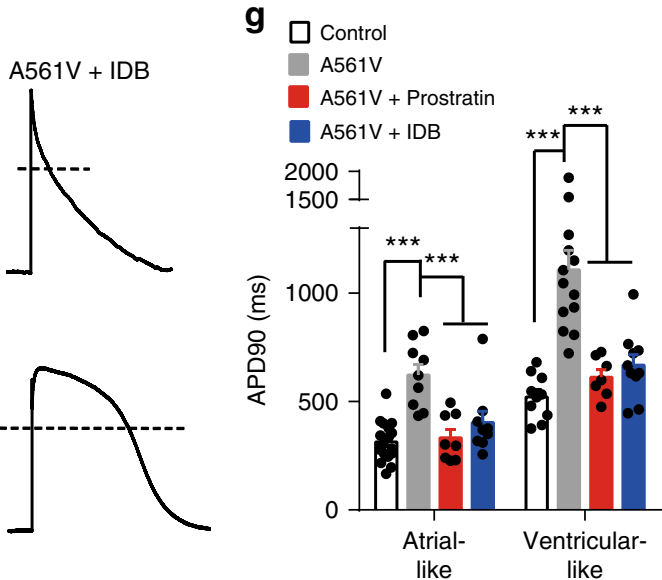

Fig. 6 Prostratin or IDB corrects electrophysiological abnormality of hiPSCA561V_CMs. a Schematic illumination of CRISPR/Cas9-mediated genome editing in $K C N H 2$ (encoding the hERG channel) locus. b Immunostaining for sarcomeric $\alpha$-actinin and Troponin I in control hiPSC-CMs and hiPSCA561V-CMs. Scale bar: $20 \mu \mathrm{m}$. c RT-PCR analysis of the cardiac-specific transcription factors (NKX2.5) and structural genes (MLC2V, MYH6, and MYH7) in control hiPSC-CM and hiPSCA561V_CMs. d Representative $l_{\mathrm{kr}}$ currents. e Tail current densities of $I_{\mathrm{kr}} . n=6,9,7$, and 7 cells for control hiPSC-CMs, hiPSCA561V_CMs, Prostratintreated hiPSC ${ }^{A 561 V}{ }_{-} C M s$, and IDB treated hiPSC ${ }^{A 561 V}{ }_{-} C M s$, respectively. $\mathbf{f}$ Representative action potentials. $\mathbf{g}$ APD90 of action potentials. $n=15,9,8$, and

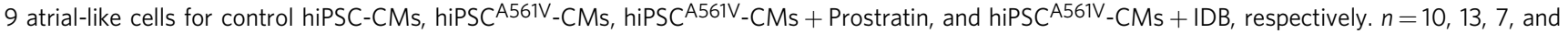
10 ventricular-like cells for control hiPSC-CMs, hiPSCA561V_CMs, hiPSCA561V_CMs + Prostratin, and hiPSCA561V_CMs + IDB, respectively. Data shown are mean \pm s.e.m. ${ }^{\star \star} P<0.01,{ }^{\star \star \star} P<0.001$ (one-way ANOVA Dunnett's test for $\mathbf{e}, \mathbf{g}$ ). All experiments were repeated at least three times

treatment of cystic fibrosis ${ }^{42}$. Some hERG blockers have been reported to promote protein trafficking of LQTS mutant channels ${ }^{26,43}$, however, the blockage action of drugs may diminish the current conducted by rescued hERG $\mathrm{K}^{+}$channels, consequently nullify their therapeutic potential. In this study, our phenotype- based screening assays in C. elegans model of LQTS led to identification of compounds that not only promote protein trafficking of LQTS mutant channels, but also correct the electrophysiological abnormality of cardiomyocytes carrying a LQTS2 mutation. Thus, the C. elegans models of LQTS provide useful 
in vivo systems for reliable identification of functional correctors of LQTS2 mutations.

Our screening has identified two hERG modulators Prostratin and IDB that are PKCe activators. The PKC signaling is known to act on cardiac sodium channels, $\mathrm{K}_{\mathrm{ATP}}$ channel, KCNQ1, and hERG in regulating the re-polarization of cardiac action potentials ${ }^{44-46}$. We found that these two compounds restored the normal function of LQTS-related mutant hERG $\mathrm{K}^{+}$channels by correcting the biogenesis defect, without affecting wild-type hERG and cardiomyocyte physiology. The PKCe may be a specific isoform of PKC that targets biogenic processing of hERG through phosphorylating the pore region of the channels. The selective and effective action of Prostratin and IDB is particularly important for their potential clinical applications. Prostratin is a natural product found in the bark of the mamala tree, and it has a potential in treatment of AIDS by specifically targeting the latent HIV reservoirs ${ }^{47,48}$. Whether Prostratin and IDB are effective for treating LQTS patients remains to be further studied.

In summary, we demonstrate that $C$. elegans models of channelopathies are excellent systems for the discovery of ion channel drugs. The robustness of this method is evidenced by the fact that we have successfully identified six compounds as functional correctors of hERG mutant channels in this study, while only about ten hERG activators have been reported previously ${ }^{49}$. Since most types of ion channels are well-conserved between C. elegans and human, particularly in the transmembrane domains where mutations for channelopathies are often found, we proposed that C. elegans models of channelopathies could be generated for other channels besides the hERG $\mathrm{K}^{+}$channel. Phenotypes of $C$. elegans channelopathy models are easy to be quantified, and hence a reasonable high-throughput screen could be done in a regular ion channel lab. Small-molecule screens in this study were done manually with about 250 drugs per person per day. Several highthroughput behavioral analysis platforms for C. elegans have been developed for different purposes ${ }^{50-52}$. With proper automation in behavioral screening, we expect that a high-throughput screening system based on our method will become an efficient tool for drug discovery for channelopathies.

\begin{abstract}
Methods
Worm strains. C. elegans strains were cultured on Nematode Growth Medium (NGM) at $20^{\circ} \mathrm{C}$. The wild-type $\mathrm{N}_{2}$ Bristol strain and the acs-20(tm3232) strain were obtained from Caenorhabditis Genetics Center and National BioResource Project, respectively. $h E R G^{\text {chimera/A536W }}$ and $h E R G^{\text {chimera/A536W/A561V }}$ transgenic worms were generated by expressing $\mathrm{P}_{\text {unc-103::hERG }}{ }^{\text {chimera/A536W }}::$ gfp and $\mathrm{P}_{\text {unc-103:: }}$ hERG ${ }^{\text {chimera/A536W/A561V }:: g f p ~ p l a s m i d s ~ i n ~} \mathrm{~N}_{2}$ worms, respectively. The $\mathrm{P}_{\text {unc-103.: }}$ mcherry plasmid was co-injected with the above plasmids to express mCherry as a cytoplasmic protein marker in these transgenic worms. The transgenic worms were integrated by the UV-TMP method ${ }^{53}$ and outcrossed for three times.
\end{abstract}

\begin{abstract}
Molecular biology. The hERG ${ }^{\text {chimera }}$ was constructed by fusing UNC-103 (isoform a) N-terminus (1-79th amino acids) with hERG transmembrane/cNBD domains (369-889th amino acids) and UNC-103 C-terminus (599-829th amino acids). PCR product of $H A-H E R G$ cDNA was subcloned into the pCI-neo vector to construct plasmids that could be expressed in mammalian cell lines. Mutations were generated by site-directed mutagenesis. All constructs were confirmed by DNA sequencing.
\end{abstract}

Immunoblot analysis. Western blot analyses were performed as described below. Briefly, $16 \mathrm{~h}$ after transfection, HEK293T cells were treated with drugs and on the next day cells were lysed in the NP-40 lysis buffer ( $1 \% \mathrm{NP}-40,150 \mathrm{mM} \mathrm{NaCl}, 1 \mathrm{mM}$ $\mathrm{NaF}, 50 \mathrm{mM}$ Tris, $\mathrm{pH}$ 7.6) supplemented with protease inhibitor mixture (05892791001, Roche). After centrifugation at $12,000 \times g$ at $4{ }^{\circ} \mathrm{C}$ for $10 \mathrm{~min}$, the supernatants were collected for Western blot analyses (full blots found in Supplementary Fig. 10). The primary antibodies used in this study were rat anti-HA (11867423001, Roche) with a dilution 1:3000, rabbit anti-PKCe (sc-214, Santa Cruz) with a dilution 1:1000, mouse anti- $\beta$-Tubulin (M30109, Abmart) with a dilution 1:3000.
Immunocytochemistry. The HEK293T cells or hiPSC-CMs were fixed with 4\% paraformaldehyde for $20 \mathrm{~min}$. After washed for three times by PBS, the fixed cells were permeabilized with $0.1 \%$ Triton X-100 for $10 \mathrm{~min}$ and then were blocked with $10 \%$ bovine serum albumin for $1 \mathrm{~h}$. For immunocytochemistry of HEK293T cells, mouse anti-HA (M20003, Abmart; 1:400), and rabbit anti-Calnexin were used as primary antibodies (ab22595, Abcam; 1:400); for immunocytochemistry of hiPSCCMs, rabbit anti-troponin I (sc-15368, Santa Cruz; 1:500) or anti-hERG (ab196301, Abcam; 1:200) and mouse anti-sarcomeric $\alpha$-actinin (A7811, Sigma; 1:600) were used as primary antibodies. Anti-mouse Alexa Fluor 594 and anti-rabbit Alexa Fluor 488-conjugated secondary antibodies were used for immunofluorescence detection.

Behavioral assays. Synchronized young adult worms were used for behavioral assays. For egg-laying assays, individual late-L4 hermaphrodite was picked on a NGM plate with E. coli strain OP50 and was picked away $24 \mathrm{~h}$ later. Total eggs and progenies in the plate were counted. The locomotion speed was measured on the NGM plate without OP50. About 25 young adult worms were picked on the plate and allowed to recover for $1 \mathrm{~min}$, and then their spontaneous movement was recorded for $1 \mathrm{~min}$. The locomotion speed was calculated by using wrMTrck software. For male's copulatory spicule protraction assays, virgin L4 males were isolated on NGM plates seeded with OP50. After $24 \mathrm{~h}$, male spicule protraction was scored if at least one spicule partially extended from the cloaca. At least 40 worms per strain were tested in each experiment. The investigators were blinded to the genotypies during $C$. elegans behavioral assays.

Small-molecule screen. Chemical libraries used in this study include the Prestwick Chemical Library, the Spectrum Collection, the Natural Products Library, the ICCB Known Bioactives Library, the IB screening Library, the NIH Clinical Collection, the FDA Approved Drug Library, the SIGMA LOPAC, and the AnalytiCon Discovery. 48-well screening plates were prepared by adding $500 \mu \mathrm{l} \mathrm{NGM}$ containing $1 \mathrm{mM}$ IPTG and $25 \mu \mathrm{g} \mathrm{ml}^{-1}$ carbenicillin in each well. A mixture of HT115 E. coli expressing double-strand RNAs targeting ifd-2 and $c 15 c 7.5$ were then seeded on NGM plates and allowed to grow for 2 days. Small molecules dissolved in DMSO were then added to NGM plates with a final concentration of $20 \mu \mathrm{M}$. After $4 \mathrm{~h}$, synchronized L4 acs-20; $h E R G^{\text {chimera } / \mathrm{A} 536 \mathrm{~W}}$ or acs-20; $h E R G^{\text {chimera } / \mathrm{A} 536 \mathrm{~W} /}$ A561V worms were rinsed from their cultivating plates and deposited into each well of these screening plates. Phenotypes of tested worms were examined $36 \mathrm{~h}$ later.

Electrophysiology. Whole-cell patch-clamp was performed by using Axon $200 \mathrm{~B}$. For recording currents in HEK293T cells expressing hERG channels, pipette solution consisted of (in mM) $135 \mathrm{KCl}, 10 \mathrm{EGTA}, 1 \mathrm{MgCl}_{2}, 5 \mathrm{Mg}$-ATP and 10 HEPES (pH 7.2 with $\mathrm{KOH}$ ), and bath solution consisted of (in $\mathrm{mM}$ ) $130 \mathrm{NaCl}, 5$ $\mathrm{KCl}, 10 \mathrm{HEPES}, 1 \mathrm{MgCl}_{2}$, 10 glucose, and $2 \mathrm{CaCl}_{2}$ (pH 7.4 with $\mathrm{NaOH}$ ). Recording was performed at room temperature by using a standard protocol that membrane potential was first pre-depolarized to $+40 \mathrm{mV}$ to inactivate the channel, and then repolarized voltage from $-140 \mathrm{mV}$ to $+40 \mathrm{mV}$ (in $20 \mathrm{mV}$ increments) to obtain tail currents.

To measure $I_{\mathrm{Kr}}$ currents and cardiac action potentials, hiPSC-CMs were cultured on $0.1 \%$ gelatin coated coverslips in a 24 -well plate. $3-8$ days after seeding, hiPSC-CMs were treated with $2 \mu \mathrm{M}$ IDB (sc-202663, Santa Cruz) or $3 \mu \mathrm{M}$ Prostratin (P0077, Sigma) for $48 \mathrm{~h}$. The $I_{\mathrm{Kr}}$ currents of hiPSC-CMs were isolated by application of hERG blocker E-4031 (M5060, Sigma). $I_{\mathrm{Kr}}$ was measured using a protocol that initially depolarizing the membrane potential from $-60 \mathrm{mV}$ to +60 $\mathrm{mV}$ in $30 \mathrm{mV}$ increments, then repolarizing to $-30 \mathrm{mV}$ to elicit tail current. The composition of the pipette solution was (in $\mathrm{mM}$ ): $120 \mathrm{KCl}, 1 \mathrm{MgCl}_{2}, 3 \mathrm{Mg}$-ATP, 10

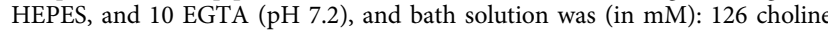
chloride, $5.4 \mathrm{KCl}, 1.8 \mathrm{CaCl}_{2}, 1 \mathrm{MgCl}_{2}, 10 \mathrm{HEPES}$, and 10 glucose (pH 7.4). Nifedipine ( $5 \mu \mathrm{M}$; N7634, Sigma) and chromanol (10 $\mu \mathrm{M}$; C2615, Sigma) were added in the bath solution to suppress potential interference of $I_{\mathrm{Ca}}$ and $I_{\mathrm{Ks}}$, respectively.

Action potentials were elicited by stimulating cardiomyocytes with depolarizing $10 \mathrm{~ms}$-impulses from $0 \mathrm{pA}$ to $40 \mathrm{pA}$ by 4 -pA increments. The frequency of stimulus for the protocol was about $0.5 \mathrm{~Hz}$. The bath solution contained (in $\mathrm{mM}$ ) $140 \mathrm{NaCl}$ $5.4 \mathrm{KCl}, 1.8 \mathrm{CaCl}_{2}, 1 \mathrm{MgCl}_{2}, 10 \mathrm{HEPES}$, and 10 glucose, $\mathrm{pH}$ 7.4. The pipette solution contained (in $\mathrm{mM}$ ) $120 \mathrm{KCl}, 1 \mathrm{MgCl}_{2}, 3 \mathrm{Mg}$-ATP, $10 \mathrm{HEPES}$, and 10 EGTA, pH 7.2.

Generation of heterozygous hERGA561V hiPSC clones. Generation of heterozygous hERG ${ }^{A 561 V}$ hiPSC clones was carried out by following a similar protocol described previously ${ }^{54}$. Briefly, wild-type 1016 hiPSC (HSCI iPS Core, Harvard) were grown in feeder-free adherent culture in chemically defined mTeSR1 (STEMCELL Technologies) supplemented with penicillin and streptomycin. Plates were precoated with Geltrex matrix (Invitrogen). The cells were disassociated into single cells with Accutase (Invitrogen), and 10 million cells were electroporated with a mix of $30 \mathrm{mg}$ of the CRISPR plasmid and $30 \mathrm{mg}$ of the donor plasmid in a single cuvette (Bio-Rad). The cells were then collected from the culture plate 48-72 $\mathrm{h}$ post-electroporation, and resuspended in PBS buffer. Cells expressing green fluorescent markers were collected by FACS (FACSAria II; BD Biosciences) and 
replated on $10 \mathrm{~cm}$ tissue-culture plates at around 20,000 cells per plate to allow for recovery in growth medium.

Post-FACS, the cells were allowed to recover for a week, and then single colonies were manually picked and replated individually to wells of 96 -well plates. Colonies were allowed to grow to near full confluence over the next 7 days, at which point they were split and replica-plated. Genomic DNA of colonies was extracted and genotyping at the CRISPR target site was identified by PCR amplication and DNA sequencing. Clones with confirmed heterozygous mutation allele or wild-type allele were expanded for further experiments.

Generation of cardiomyocytes from hiPSC. Generation of cardiomyocytes from hiPSC was performed by following the previously reported method ${ }^{55}$ with modifications. Cardioeasy chemically defined cardiac differentiation kit (CA2004500, Cellapy) was used for differentiation hiPSC into cardiomyocytes. Briefly, human hiPSC were splitted using $0.5 \mathrm{mM}$ EDTA PBS solution at 1:10 ratio and cultured with PSCeasy chemically defined medium for 3 days. When reached $\sim 80 \%$ confluence, hiPSC was cultured in Induction Medium II for 2 days and then in Induction Medium III. Induction Medium III was changed every other day afterwards. Spontaneously contracting cells were observed from the day 8 after induction. The derived cardiomyocytes were purified with Cardioeasy purification medium at the day 15 after induction.

Statistical analysis. Statistical analysis was done by using GraphPad Prism 6.0. Data shown are mean \pm s.e.m. For the C. elegans behavior data, unpaired Student's $t$-test was used to determine significant differences between samples. For electrophysiological data, one-way ANOVA Dunnett's test was used when comparing multiple groups. Significance levels: ${ }^{*} P<0.05,{ }^{*} P<0.01,{ }^{* * *} P<0.001$. The ALA inhibition curve was fitted with equation: $y(x)=y_{\min }+\left(y_{\max }-y_{\min }\right) /\left(1+10^{(x-}\right.$ $\log (\mathrm{C} 50)$ ). Where $x$ is the log of concentration; $y(x)$ is response, decreasing as $x$ increase; IC50, the median inhibitory concentration. The stimulation curve of Prostratin or IDB on hERG ${ }^{\mathrm{A} 561 \mathrm{~V}}$ current densities was performed with Gaussian fit. No statistical method was used to predetermine sample size. All experiments were repeated at least for three times.

\section{Data availability}

All data supporting the findings of this study are included in this article (and its Supplementary Information files). Further data are available from the corresponding author upon reasonable request.

Received: 20 March 2018 Accepted: 31 August 2018

Published online: 26 September 2018

\section{References}

1. Kim, J. B. Channelopathies. Korean J. Pediatr. 57, 1-18 (2014).

2. Farre, C. \& Fertig, N. New strategies in ion channel screening for drug discovery: are there ways to improve its productivity? Expert Opin. Drug Discov. 9, 1103-1107 (2014).

3. McManus, O. B. HTS assays for developing the molecular pharmacology of ion channels. Curr. Opin. Pharmacol. 15, 91-96 (2014).

4. Isom, L. L., De Jongh, K. S. \& Catterall, W. A. Auxiliary subunits of voltagegated ion channels. Neuron 12, 1183-1194 (1994).

5. Ashcroft, F. M. From molecule to malady. Nature 440, 440-447 (2006).

6. Garcia, L. R. \& Sternberg, P. W. Caenorhabditis elegans UNC-103 ERG-like potassium channel regulates contractile behaviors of sex muscles in males before and during mating. J. Neurosci. 23, 2696-2705 (2003).

7. Park, E. C. \& Horvitz, H. R. Mutations with dominant effects on the behavior and morphology of the nematode Caenorhabditis elegans. Genetics 113, 821-852 (1986).

8. Strange, K. From genes to integrative physiology: ion channel and transporter biology in Caenorhabditis elegans. Physiol. Rev. 83, 377-415 (2003).

9. Goodman, M. B. et al. MEC-2 regulates C. elegans $\mathrm{DEG} / \mathrm{ENaC}$ channels needed for mechanosensation. Nature 415, 1039-1042 (2002)

10. Bianchi, L., Kwok, S. M., Driscoll, M. \& Sesti, F. A potassium channel-MiRP complex controls neurosensory function in Caenorhabditis elegans. J. Biol. Chem. 278, 12415-12424 (2003).

11. Chen, X., Ruan, M. Y. \& Cai, S. Q. KChIP-like auxiliary subunits of Kv4 channels regulate excitability of muscle cells and control male turning behavior during mating in Caenorhabditis elegans. J. Neurosci. 35, 1880-1891 (2015).

12. Petersen, C. I. et al. In vivo identification of genes that modify ether-a-go-gorelated gene activity in Caenorhabditis elegans may also affect human cardiac arrhythmia. Proc. Natl Acad. Sci. USA 101, 11773-11778 (2004).

13. $\mathrm{Li}, \mathrm{K}$. et al. Tetrameric assembly of $\mathrm{K}+$ channels requires ER-located chaperone proteins. Mol. Cell 65, 52-65 (2017).
14. Kwok, T. C. et al. A small-molecule screen in C. elegans yields a new calcium channel antagonist. Nature 441, 91-95 (2006).

15. Kaletta, T. \& Hengartner, M. O. Finding function in novel targets: C. elegans as a model organism. Nat. Rev. Drug Discov. 5, 387-398 (2006).

16. Burns, A. R. et al. A predictive model for drug bioaccumulation and bioactivity in Caenorhabditis elegans. Nat. Chem. Biol. 6, 549-557 (2010).

17. Warmke, J. W. \& Ganetzky, B. A family of potassium channel genes related to eag in Drosophila and mammals. Proc. Natl Acad. Sci. USA 91, 3438-3442 (1994).

18. Curran, M. E. et al. A molecular basis for cardiac arrhythmia: HERG mutations cause long QT syndrome. Cell 80, 795-803 (1995).

19. Sanguinetti, M. C., Jiang, C., Curran, M. E. \& Keating, M. T. A mechanistic link between an inherited and an acquired cardiac arrhythmia: HERG encodes the IKr potassium channel. Cell 81, 299-307 (1995).

20. Trudeau, M. C., Warmke, J. W., Ganetzky, B. \& Robertson, G. A. HERG, a human inward rectifier in the voltage-gated potassium channel family. Science 269, 92-95 (1995)

21. Abbott, G. W. et al. MiRP1 forms IKr potassium channels with HERG and is associated with cardiac arrhythmia. Cell 97, 175-187 (1999).

22. Vandenberg, J. I. et al. hERG K(+) channels: structure, function, and clinical significance. Physiol. Rev. 92, 1393-1478 (2012).

23. Schwartz, P. J. et al. Prevalence of the congenital long-QT syndrome. Circulation 120, 1761-1767 (2009).

24. Sanguinetti, M. C. \& Tristani-Firouzi, M. hERG potassium channels and cardiac arrhythmia. Nature 440, 463-469 (2006).

25. Anderson, C. L. et al. Large-scale mutational analysis of Kv11.1 reveals molecular insights into type 2 long QT syndrome. Nat. Commun. 5, 5535 (2014).

26. Anderson, C. L. et al. Most LQT2 mutations reduce Kv11.1 (hERG) current by a class 2 (trafficking-deficient) mechanism. Circulation 113, 365-373 (2006).

27. Mehta, A. et al. Re-trafficking of hERG reverses long QT syndrome 2 phenotype in human iPS-derived cardiomyocytes. Cardiovasc. Res. 102, 497-506 (2014).

28. Zhou, Z., Gong, Q. \& January, C. T.Correction of defective protein trafficking of a mutant HERG potassium channel in human long QT syndrome. Pharmacological and temperature effects. J. Biol. Chem. 274, 31123-31126 (1999).

29. Salkoff, L. et al. Analysis of C. elegans genes required for maintaining intestinal lumen morphology and ingested drug resistance. WormBook: The Online Review of C. elegans Biology, 1-15 (Wormbook, Pasadena, CA, 2005)

30. Subbiah, R. N., Kondo, M., Campbell, T. J. \& Vandenberg, J. I. Tryptophan scanning mutagenesis of the HERG $\mathrm{K}+$ channel: the $\mathrm{S} 4$ domain is loosely packed and likely to be lipid exposed. J. Physiol. 569, 367-379 (2005).

31. Kage-Nakadai, E. et al. Two very long chain fatty acid acyl-CoA synthetase genes, acs-20 and acs-22, have roles in the cuticle surface barrier in Caenorhabditis elegans. PLoS ONE 5, e8857 (2010).

32. Paulson, C. C. 220 pages (Southern Methodist University, Dallas, TX, 2009).

33. Curran, J. \& Mohler, P. J. Alternative paradigms for ion channelopathies: disorders of ion channel membrane trafficking and posttranslational modification. Annu. Rev. Physiol. 77, 505-524 (2015).

34. Harkcom, W. T. \& Abbott, G. W. Emerging concepts in the pharmacogenomics of arrhythmias: ion channel trafficking. Expert Rev. Cardiovasc. Ther. 8, 1161-1173 (2010).

35. Kagan, A., Yu, Z., Fishman, G. I. \& McDonald, T. V. The dominant negative LQT2 mutation A561V reduces wild-type HERG expression. J. Biol. Chem. 275, 11241-11248 (2000)

36. Ficker, E. et al. Retention in the endoplasmic reticulum as a mechanism of dominant-negative current suppression in human long QT syndrome. J. Mol. Cell. Cardiol. 32, 2327-2337 (2000).

37. Thomas, D. et al. Regulation of HERG potassium channel activation by protein kinase $\mathrm{C}$ independent of direct phosphorylation of the channel protein. Cardiovasc. Res. 59, 14-26 (2003).

38. Itzhaki, I. et al. Modelling the long QT syndrome with induced pluripotent stem cells. Nature 471, 225-229 (2011)

39. Kullmann, D. M. \& Waxman, S. G. Neurological channelopathies: new insights into disease mechanisms and ion channel function. J. Physiol. 588, 1823-1827 (2010)

40. Gao, Y., Yechikov, S., Vazquez, A. E., Chen, D. \& Nie, L. Impaired surface expression and conductance of the KCNQ4 channel lead to sensorineural hearing loss. J. Cell Mol. Med. 17, 889-900 (2013).

41. Rajakulendran, S., Schorge, S., Kullmann, D. M. \& Hanna, M. G. Episodic ataxia type 1: a neuronal potassium channelopathy. Neurotherapeutics 4 , 258-266 (2007)

42. Brodsky, J. L. \& Frizzell, R. A. A combination therapy for cystic fibrosis. Cell 163, 17 (2015).

43. Ficker, E., Dennis, A. T., Wang, L. \& Brown, A. M. Role of the cytosolic chaperones Hsp70 and Hsp90 in maturation of the cardiac potassium channel HERG. Circ. Res. 92, e87-e100 (2003). 
44. Krishnan, Y., Li, Y., Zheng, R., Kanda, V. \& McDonald, T. V. Mechanisms underlying the protein-kinase mediated regulation of the HERG potassium channel synthesis. Biochim. Biophys. Acta 1823, 1273-1284 (2012).

45. Radresa, O., Guia, A. \& Baroudi, G. Roles of PKC isoforms in PMA-induced modulation of the hERG channel (Kv11.1). J. Biomol. Screen. 19, 890-899 (2014).

46. Ferreira, J. C., Mochly-Rosen, D. \& Boutjdir, M. Regulation of cardiac excitability by protein kinase C isozymes. Front. Biosci. 4, 532-546 (2012).

47. Gulakowski, R. J., McMahon, J. B., Buckheit, R. W. Jr., Gustafson, K. R. \& Boyd, M. R. Antireplicative and anticytopathic activities of prostratin, a nontumor-promoting phorbol ester, against human immunodeficiency virus (HIV). Antivir. Res. 33, 87-97 (1997).

48. Gustafson, K. R. et al. A nonpromoting phorbol from the samoan medicinal plant Homalanthus nutans inhibits cell killing by HIV-1. J. Med. Chem. 35, 1978-1986 (1992).

49. Zhou, P. Z., Babcock, J., Liu, L. Q., Li, M. \& Gao, Z. B. Activation of human ether-a-go-go related gene (hERG) potassium channels by small molecules. Acta Pharmacol. Sin. 32, 781-788 (2011).

50. Swierczek, N. A., Giles, A. C., Rankin, C. H. \& Kerr, R. A. High-throughput behavioral analysis in C. elegans. Nat. Methods 8, 592-598 (2011).

51. Mathew, M. D., Mathew, N. D. \& Ebert, P. R. WormScan: a technique for high-throughput phenotypic analysis of Caenorhabditis elegans. PLoS ONE 7, e33483 (2012).

52. O’Reilly, L. P., Luke, C. J., Perlmutter, D. H., Silverman, G. A. \& Pak, S. C. C. elegans in high-throughput drug discovery. Adv. Drug Deliv. Rev. 69-70, 247-253 (2014).

53. Yin, J. A., Liu, X. J., Yuan, J., Jiang, J. \& Cai, S. Q. Longevity manipulations differentially affect serotonin/dopamine level and behavioral deterioration in aging Caenorhabditis elegans. J. Neurosci. 34, 3947-3958 (2014).

54. Ding, Q. et al. Enhanced efficiency of human pluripotent stem cell genome editing through replacing TALENs with CRISPRs. Cell Stem Cell 12, 393-394 (2013).

55. Lan, F. et al. Abnormal calcium handling properties underlie familial hypertrophic cardiomyopathy pathology in patient-specific induced pluripotent stem cells. Cell Stem Cell 12, 101-113 (2013).

\section{Acknowledgements}

We thank Mu-ming Poo and Ying Liu for critical reading of the manuscript, Mei-Yu Ruan for technical assistance, the C. elegans Genetics Center (funded by NIH Office of Research Infrastructure Programs P40 OD010440) and S. Mitani (Tokyo Women's Medical University School of Medicine) for providing us strains and National Compound Resource Center for providing us small-molecule libraries. This work was supported by grants to S.-Q.C. (the Strategic Priority Research Program of Chinese Academy of Science, XDBS1020100 and the National Natural Science Foundation of China, 31471149 and 81527901), to F.L. (the National Natural Science Foundation of China,
81422003), and to Q.D. (the National Key R\&D Program of China, 2017YFA0102800 and 2017YFA0103700).

\section{Author contributions}

Q.J., K.L. and S.-Q.C. designed all the experiments. Q.J. and K.L. performed most of the experiments. Q.J. generated the C. elegans models of channelopathies, performed small-molecule screening assays, and the electrophysiological experiments. K.L. performed the biochemical assays. W.-J.L. conducted the cardiomyocyte differentiation of the hiPSCs. S.L. and Q.J. generated the CRISPR/Cas9-edited hiPSC clones. X.C. performed the electrophysiological recordings of some of the hERG mutant channels expressed in HEK293T cells. J.Y. and X.-J.L. assisted in carrying out the small-molecule screenings. Q.J. and K. L. performed the behavioral assays. F.L. supervised the cardiomyocyte differentiation experiments and analyzed the data. Q.D. supervised the CRISPR/ Cas9 editing experiments and analyzed the data. Q.J., K.L., F.L., Q.D. and S.-Q.C. prepared the figures and wrote the manuscript.

\section{Additional information}

Supplementary Information accompanies this paper at https://doi.org/10.1038/s41467018-06514-5.

Competing interests: W.-J.L is a cofounder of Beijing Cellapy Biological Technology Co., Ltd. The remaining authors declare no competing interests.

Reprints and permission information is available online at http://npg.nature.com/ reprintsandpermissions/

Publisher's note: Springer Nature remains neutral with regard to jurisdictional claims in published maps and institutional affiliations.

\begin{abstract}
(c) (i) Open Access This article is licensed under a Creative Commons cc) Attribution 4.0 International License, which permits use, sharing, adaptation, distribution and reproduction in any medium or format, as long as you give appropriate credit to the original author(s) and the source, provide a link to the Creative Commons license, and indicate if changes were made. The images or other third party material in this article are included in the article's Creative Commons license, unless indicated otherwise in a credit line to the material. If material is not included in the article's Creative Commons license and your intended use is not permitted by statutory regulation or exceeds the permitted use, you will need to obtain permission directly from the copyright holder. To view a copy of this license, visit http://creativecommons.org/ licenses/by/4.0/.
\end{abstract}

(C) The Author(s) 2018 